\title{
Probability distributions of bed load transport rates: A new derivation and comparison with field data
}

\begin{abstract}
Jens M. Turowski ${ }^{1}$
Received 5 August 2009; revised 16 February 2010; accepted 24 February 2010; published 3 August 2010.

[1] It has been known for a long time that sediment transport rates can vary strongly even if the ambient hydraulic conditions remain steady. In this article, a new approach is described to derive probability distributions of bed load transport rates, starting from the waiting time between the arrivals of individual sediment particles. The formalism should be valid when transport is not dominated by bed form motion. Without any assumptions about the distribution of interarrival times, the approach yields the Birnbaum-Saunders distribution, a two-parameter distribution previously used in lifetime modeling. Observed dependence of mean transport rates on the sampling time and multiscaling are predicted by the distribution. Assuming exponentially and Poisson-distributed interarrival times, the same approach yields the Poisson and the gamma distributions. Using a high-resolution bed load transport data set from the Pitzbach, Austria, the distribution functions are tested on field data. The gamma distribution best describes the data, with maximum deviations of $\sim 5 \%$. However, the Birnbaum-Saunders distribution may be more useful in certain applications, as it is a general approximation in the proposed formalism and no debated assumptions are necessary for its derivation.
\end{abstract}

Citation: Turowski, J. M. (2010), Probability distributions of bed load transport rates: A new derivation and comparison with field data, Water Resour. Res., 46, W08501, doi:10.1029/2009WR008488.

\section{Introduction}

[2] Bed load transport, the water-driven rolling, sliding, or hopping motion of coarse particles in a stream, is an important mode of sediment transfer in rivers and a key process in shaping the Earth's surface. Accurate calculation of transport rates is necessary both in engineering applications such as flood hazard mitigation and in pure science. Bed load transport rates are known to fluctuate strongly both in nature [e.g., Gomez and Church, 1989; Hassan and Church, 2000] and in the laboratory [e.g., Kuhnle and Southard, 1988; Ancey et al., 2008] even under steady flow conditions. Several different causes for these fluctuations have been identified [Gomez et al., 1989; Hoey, 1992], including variations in sediment supply [e.g., Benda and Dunne, 1997], spatially and temporally varying distribution of grain sizes, relative grain arrangement, and grain sorting processes [e.g., Kirchner et al., 1990; Chen and Stone, 2008], and the passage of bed forms [e.g., Lisle et al., 2001; Recking et al., 2009].

[3] Several models have been proposed to describe the probability distribution functions of bed load transport rates. Einstein [1937] considered bed load transport as a series of rest periods of random length, interrupted by short periods of motion of random distances. He assumed that both step lengths and rest times are exponentially distributed and

\footnotetext{
${ }^{1}$ Eidgenössische Forschungsanstalt WSL Birmensdorf, Birmensdorf, Switzerland.
}

Copyright 2010 by the American Geophysical Union. 0043-1397/10/2009WR008488 derived distribution functions for the amount of sediment transported over a cross section. However, it is difficult to measure the distribution functions of rest periods and transport distances directly in the field or laboratory and many of the assumptions underlying Einstein's [1937] model have not yet been validated. Guided by laboratory observations, Hamamori [1962] developed a distribution function of bed load transport rates for cases when bed form motion is dominant. In his model, secondary dunes are responsible for the total transport. These secondary dunes entrain material and grow linearly with distance while moving up the stoss slope of primary dunes. Thus, the assumptions underlying his distribution are physically restrictive and apply only in limited circumstances. More recent stochastic models of bed load transport often describe the entrainment and deposition of particles in a control volume using Markov birth-death models [e.g., Lisle et al., 1998; Papanicolaou et al., 2002; Ancey et al., 2006, 2008; Turowski, 2009]. These models typically feature a large number of parameters that need to be calibrated on data but are hard to measure even under controlled laboratory conditions [cf. Ancey et al., 2008]. Thus, it is challenging to test and validate such models directly.

[4] Here I take a different approach. Instead of trying to devise an accurate description of the physics of bed load transport, I consider the distribution of waiting times between particle arrivals (interarrival times) at a cross section to derive probability distributions for bed load transport rates. Using modern equipment such as light tables [Frey et al., 2003; Zimmermann et al., 2008] or video cameras [Drake et al., 1988], it should be possible to directly measure this distribution in the laboratory and the field. The 
Birnbaum-Saunders distribution [Birnbaum and Saunders, 1968] arises as a general approximation when no explicit assumptions about the distribution of waiting times are made. By assuming exponentially or Poisson distributed waiting times, one arrives at the Poisson and Gamma distributions, respectively. The distribution functions are compared to a large field data set from the Pitzbach, Austria [Rickenmann and McArdell, 2008; Turowski and Rickenmann, 2009].

\section{Distribution Functions of Bed Load Transport Rates}

\subsection{The Birnbaum-Saunders Distribution}

[5] In practice often the sediment flux at a channel cross section is of interest. Particles arrive at varying intervals, and we want to know the total sediment volume arriving within a certain time period and its variability. For the derivation of the distribution function, I make the following formal assumptions:

[6] 1. The waiting time between the arrivals of individual bed load particles (interarrival time) at a cross section is a stochastic variable with an unspecified distribution with well-defined mean $\mu$ and variance $\sigma_{\mathrm{w}}^{2}$.

[7] 2. Within each measurement interval, enough particles arrive such that the central limit theorem and the law of large numbers are applicable.

[8] Implicit in these two assumptions is the notion that the particles are actually countable and that individual arrivals are statistically independent. The following derivation may thus not be applicable to environments where transport by bed form motion is dominant. This constraint and the two assumptions are discussed in more detail in section 4.1.

[9] Consider the arrival of individual sediment particles at the measurement cross section under steady conditions. The interarrival time is described by a random variable $\tau$ with mean $\mu$ and variance $\sigma_{\mathrm{w}}^{2}$. The time $T_{N}$ at the arrival of the $N^{\text {th }}$ particle is then

$$
T_{N}=T_{N-1}+\tau_{N}
$$

Assuming that there are a large number of particles, the central limit theorem may be applied. Hence, the probability density function (pdf) for the total time $T_{N}$ (with corresponding stochastic variable $t_{N}$ ) at the arrival of the $N^{\text {th }}$ particle is approximately normal.

$$
\operatorname{pdf}\left(T_{N}(N)\right) \approx \frac{1}{\sigma_{\mathrm{w}} \sqrt{2 \pi N}} \exp \left\{-\frac{\left(t_{N}-N \mu\right)^{2}}{2 N \sigma_{\mathrm{w}}^{2}}\right\} .
$$

Note that the normal distribution in equation (2) is not assumed but arises as a general approximation from the central limit theorem. So far, no assumptions have been made on the underlying distribution of interarrival times. The size of particles and thus their mass follow a certain site-specific distribution. Since $N$ is large, the law of large numbers applies and the total mass is $m \approx N \bar{m}$, where $\bar{m}$ is the mean mass of a single particle. Hence, the cumulative distribution function $\operatorname{cdf}\left(t_{N}\right)$ of $T_{N}$ is the cumulative normal distribution $\Phi((x-\mu) / \sigma)$ of the random variable $x$ with mean $\mu$ and standard deviation $\sigma$, which is given by

$$
\begin{aligned}
\operatorname{cdf}(t) & =P\left(T_{N}(m) \leq t_{N}\right) \\
& =\int_{-\infty}^{m} \frac{1}{\sigma_{\mathrm{w}} \sqrt{2 \pi^{m / m}}} \exp \left\{-\frac{\bar{m}\left(t_{N}-\frac{m \mu}{\bar{m}}\right)^{2}}{2 m \sigma_{\mathrm{w}}^{2}}\right\} d t \\
& =\Phi\left(\frac{t_{N}-\frac{m \mu}{\bar{m}}}{\sqrt{\frac{m}{\bar{m}}} \sigma_{\mathrm{w}}}\right) .
\end{aligned}
$$

The event $\left\{M\left(t_{N}\right) \leq m\right\}$ is equivalent to the event $\left\{T_{N}(m) \geq t_{N}\right\}$, where $M\left(t_{N}\right)$ is the random variable representing the mass at time $t_{N}$. The $\operatorname{cdf}(m)$ of $M$ at given $t_{N}$ is then

$$
\operatorname{cdf}(m)=P(M(t) \leq m)=1-P(T(m) \leq t)=\Phi\left(\frac{\frac{m \mu}{\bar{m}}-t}{\sqrt{\frac{m}{\bar{m}} \sigma_{\mathrm{w}}}}\right)
$$

where $t_{N}$ has now been replaced by a constant $t$ denoting the measurement interval. The probability density function is

$$
\operatorname{pdf}(m \mid t)=\frac{\mathrm{d} G(m)}{\mathrm{d} m}=\frac{\mu \frac{m}{\bar{m}}+t}{2 \sigma_{\mathrm{w}} m \sqrt{2 \pi \frac{m}{\bar{m}}}} \exp \left\{-\frac{\bar{m}\left(\mu \frac{m}{\bar{m}}-t\right)^{2}}{2 m \sigma_{\mathrm{w}}^{2}}\right\} .
$$

Reparameterizing with $\beta=\bar{m} t / \mu$ and $\gamma^{2}=\sigma_{\mathrm{w}}^{2} / \mu t$ to get the standard form of the Birnbaum-Saunders distribution yields

$$
\operatorname{pdf}(m \mid t)=\frac{m+\beta}{2 \gamma m \sqrt{2 \pi \beta m}} \exp \left\{-\frac{(m-\beta)^{2}}{2 m \beta \gamma^{2}}\right\} .
$$

The Birnbaum-Saunders distribution has previously been proposed to model the times at failure due to fatigue of material under cyclic stresses and belongs to a two-parameter exponential family [Birnbaum and Saunders, 1968]. The formalism of the derivation given above closely follows the one developed by Desmond [1985].

[10] Equation (6) can easily be rewritten in terms of fluxes (defined as $Q_{\mathrm{s}}=\mathrm{m} / \mathrm{t}$ ) and the pdf of the bed load transport rate $Q_{\mathrm{s}}$ in a given measurement interval $t$ is given by

$$
\operatorname{pdf}\left(Q_{\mathrm{s}} \mid t\right)=\frac{Q_{\mathrm{s}}+\beta^{\prime}}{2 \gamma Q_{\mathrm{s}} \sqrt{2 \pi \beta^{\prime} Q_{\mathrm{s}}}} \exp \left\{-\frac{\left(Q_{\mathrm{s}}-\beta^{\prime}\right)^{2}}{2 Q_{\mathrm{s}} \beta^{\prime} \gamma^{2}}\right\} .
$$

Here $\beta^{\prime}=\bar{m} / \mu$ is a scale parameter, and $\gamma^{2}=\sigma_{\mathrm{w}}^{2} / \mu t$ is a shape parameter as before. The expectation value $\mathrm{E}\left(Q_{\mathrm{s}}\right)$ of equation (7) is given by

$$
E\left(Q_{\mathrm{s}}\right)=\beta^{\prime}\left(1+\frac{\gamma^{2}}{2}\right)=\bar{m}\left(\frac{1}{\mu}+\frac{1}{2} \frac{\sigma_{\mathrm{w}}^{2}}{t \mu^{2}}\right) .
$$

And the variance $\operatorname{var}\left(Q_{\mathrm{s}}\right)$ is given by

$$
\operatorname{var}\left(Q_{s}\right)=\gamma^{2} \beta^{\prime 2}\left(1+\frac{5 \gamma^{2}}{4}\right)=\frac{\bar{m}^{2} \sigma^{2}}{\mu^{2}}\left(\frac{1}{\mu t}+\frac{5}{4} \frac{\sigma_{\mathrm{w}}^{2}}{t^{2} \mu^{2}}\right) .
$$


The standard deviation $\operatorname{std}\left(Q_{\mathrm{s}}\right)$ is equal to the square root of $\operatorname{var}\left(Q_{\mathrm{s}}\right)$. More information on the properties of the distribution can be found in the work of Johnson et al. [1995]. As would be expected, the expectation value $\mathrm{E}\left(Q_{\mathrm{s}}\right)$ approaches the constant value $\bar{m} / \mu$ as $t$ goes to infinity. This gives a constraint on the minimum length of the measurement interval $t$ : it needs to be much larger than $\bar{m} \sigma_{\mathrm{w}}^{2} / 2 \mu^{2}$ to obtain a reliable estimate of transport rates which is independent of the length of the measurement interval and thus comparable for different streams or for the same stream at different discharges [cf. Singh et al., 2009].

[11] The Birnbaum-Saunders distribution was derived without assumptions about the probability distribution of interarrival times. I will now outline two additional derivations, assuming interarrival times, which are distributed according to the exponential distribution and a continuous form of the Poisson distribution. It is not my aim here to argue that either of these distributions is the correct one, although at least the exponential distribution has been predicted from certain physically based stochastic transport theories [e.g., Ancey et al., 2008]. Rather, I want to show (1) how physically based results can be incorporated into the framework developed above and (2) how such assumptions can yield various plausible probability distributions for bed load transport rates.

\subsection{Example 1: Exponentially Distributed Interarrival Times}

[12] Let the interarrival times be exponentially distributed with a pdf of the following form:

$$
\operatorname{pdf}(\tau)=\frac{1}{\mu} \exp \left\{-\frac{\tau}{\mu}\right\}
$$

The time at the $N^{\text {th }}$ arrival is then distributed according to the gamma distribution

$$
\operatorname{pdf}\left(T_{N}(N)\right)=\left(\frac{1}{\mu}\right)^{N} \frac{t_{N}^{N-1}}{\Gamma(N)} \exp \left\{-\frac{t_{N}}{\mu}\right\} .
$$

The gamma function $\Gamma(x)$ is defined by

$$
\Gamma(x)=\int_{0}^{\infty} z^{x-1} e^{-z} \mathrm{~d} z .
$$

Following the remaining steps of the derivation as given above one obtains the cumulative distribution function (cdf) of masses

$$
\operatorname{cdf}(m)=\frac{\Gamma\left(\frac{m}{\bar{m}}, \frac{t}{\mu}\right)}{\Gamma\left(\frac{m}{\bar{m}}\right)} .
$$

Here the upper incomplete gamma function $\Gamma(x, y)$ is defined by

$$
\Gamma(x, y)=\int_{y}^{\infty} z^{x-1} e^{-z} \mathrm{~d} z
$$

Equation (13) is a continuous form of the Poisson distribution with the following pdf:

$$
\operatorname{pdf}(m)=\frac{\exp \left\{-\frac{t}{\mu}\right\}}{\Gamma\left(\frac{m}{\bar{m}}\right)}\left(\frac{t}{\mu}\right)^{m / \bar{m}} .
$$

Here the usual factorial is replaced by the gamma function (equation (12)), which is a continuous interpolation of the factorial function. This generalizes the Poisson distribution to apply for continuous variables. In the remainder of the article whenever a Poisson distribution is mentioned, I generally refer to a continuous version analogous to equation (15).

\subsection{Example 2: Interarrival Times Distributed After a Continuous Version of the Poisson Distribution}

[13] Alternatively, let the interarrival times be distributed after a continuous form of the Poisson distribution with a pdf analogous to equation (15)

$$
\operatorname{pdf}(\tau)=\frac{1}{\mu} \frac{\exp \{-1\}}{\Gamma(\tau / \mu)} 1^{\tau / \mu} .
$$

Here the expected number of occurrences is equal to one, because the interarrival time $\tau$ was normalized by the mean $\mu$. Note that this normalization of $\tau$ with $\mu$ is necessary to keep the equation dimensionally consistent. Of course, the term $1^{\tau / \mu}$ evaluates to one; I left it in to make the connection to equations (15) and (17) more explicit. The time at the $N^{\text {th }}$ arrival is also based on a continuous Poisson distribution

$$
\operatorname{pdf}\left(T_{N}(N)\right)=\frac{1}{\mu} \frac{\exp \{-N\}}{\Gamma\left(t_{N} / \mu\right)} N^{t_{N} / \mu} .
$$

Following through the remaining steps of the derivation, the masses are then distributed according to the cdf

$$
\operatorname{cdf}(m)=\frac{1-\Gamma\left(\frac{t}{\mu}, \frac{m}{\bar{m}}\right)}{\Gamma(t / \mu)} .
$$

Equation (18) gives the cdf for the gamma distribution, and the corresponding pdf is

$$
\operatorname{pdf}(m)=\left(\frac{1}{\bar{m}}\right)^{\frac{t}{\mu}} \frac{m^{t / \mu}-1}{\Gamma(t / \mu)} \exp \left\{-\frac{m}{\bar{m}}\right\} .
$$

The Birnbaum-Saunders distribution (equation (7)) is an approximation for both the continuous Poisson distribution (equation (15)) and the gamma distribution (equation (19)), which works well for large transport rates.

\section{Testing the Distributions: The Pitzbach Sediment Transport Observations}

\subsection{Field Site: Pitzbach, Austria}

[14] The Pitzbach is a small glacially fed stream in southwestern Austria near the village of Imst on the southern side of the Inn valley (Tyrol). It is a gravel bed river with a 


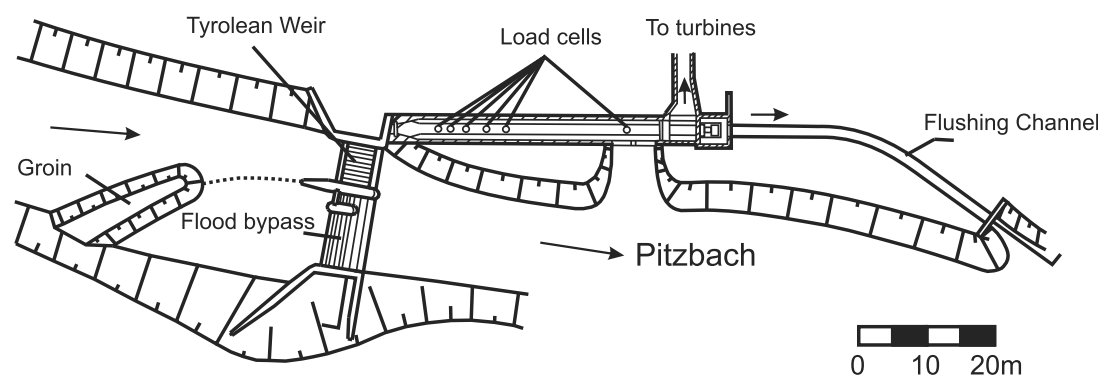

Figure 1. Schematic plan of the weir and water intake at the Pitzbach. The water flows through a Tyrolean weir with a grid size of $15 \mathrm{~cm}$ and is led into the sediment settling basin on the orographic left. There, several load cells are used to monitor the deposited volumes every $15 \mathrm{~min}$. If a threshold weight is exceeded, the basin is automatically flushed and the sediment is reinserted into the channel downstream of the weir.

median grain size of $3-4 \mathrm{~cm}$ on the bed surface and an average channel bed slope of $\sim 9 \%$. At an elevation of $1811 \mathrm{~m}$ asl the Tyrolean Water Company (TIWAG) maintains a water intake in the stream for a hydro power plant (Figure 1). Approximately $60 \%$ of the total drainage area of $26.8 \mathrm{~km}^{2}$ is covered by glaciers. As a result, most bed load is transported throughout the summer months, when discharge varies with the daily cyclicity typical for glaciated catchments. At the water intake, the water flows over a Tyrolean wear consisting of a metal grid with a bar spacing of $15 \mathrm{~cm}$ into a sediment settling basin. For the summers 1994 and 1995, the basin was equipped with five load cells and a pressure sensor, which were used to measure the amount of accumulated sediment every $15 \mathrm{~min}$. When a threshold weight was exceeded, the sediment in the basin was automatically flushed out and reinserted into the channel downstream of the weir. Since discharge is largely melt-water driven, it generally varies slowly over time and the assumption that discharge is constant within a measurement interval can be made. More information on the stream, the instruments and the measurement campaign can be found in the works of Hofer [1987], Rickenmann and McArdell [2008], and Turowski and Rickenmann [2009]. For the survey period more than 10,600 measurements with nonzero bed load transport rates are available (Figure 2). The instruments reliably pick up a signal when the deposited volume exceeds $0.05 \mathrm{~m}^{3}$. Smaller volumes are often missed and are thus underrepresented in the measurements. At a given discharge, measured transport rates scatter over up to four orders of magnitude around the mean. Nonlinear regression as often used to analyze bed load transport measurements neglects this dimension and thus is inadequate to provide a full description of the data.

\subsection{The Pitzbach Data and the Birnbaum-Saunders Distribution}

[15] The Pitzbach measurements were classified into logarithmically distributed bins in discharge, and equation (7) was fitted to the data within each bin using maximum likelihood estimation (Figure 3). Such a method is preferable to nonlinear regression as commonly used to analyze bed load transport data, since it explicitly acknowledges the spread of transport rates at a given discharge and allows the investigation of statistics other than the mean. For the Pitzbach data, the tail behavior at large transport rates is well described by the Birnbaum-Saunders distribution in all cases; however, for small transport rates there are systematic differences: at very small and medium transport rates equation (7) underpredicts the occurrence probability, for small rates it overpredicts it.

[16] At the Pitzbach, both the expected value $\mathrm{E}\left(Q_{\mathrm{s}}\right)$ and the standard deviation $\operatorname{std}\left(Q_{\mathrm{s}}\right)$ are monotonically increasing functions of discharge, well described by either an exponential or a power function (Figures 2 and 4). However, the good fit featured by the exponential function may be misleading, as for low-transport rates, an important fraction of the data may be cut off due the measurement threshold of the instruments, which often miss small deposited values (Figure 2).

[17] For discharge classes with a large number of data points $(>80)$ the coefficient of variation $\operatorname{cva}\left(Q_{\mathrm{s}}\right)=\operatorname{std}\left(Q_{\mathrm{s}}\right) /$ $\mathrm{E}\left(Q_{\mathrm{s}}\right)$ is approximately constant with an average value of 1.2. Similarly, Willis and Bolton [1979] observed cva $\left(Q_{\mathrm{s}}\right)$ $\sim 1.6$ for experimental sediment transport data of sand, and Kuhnle and Willis [1998] reported approximately equal mean and standard deviation at a given shear stress (which is

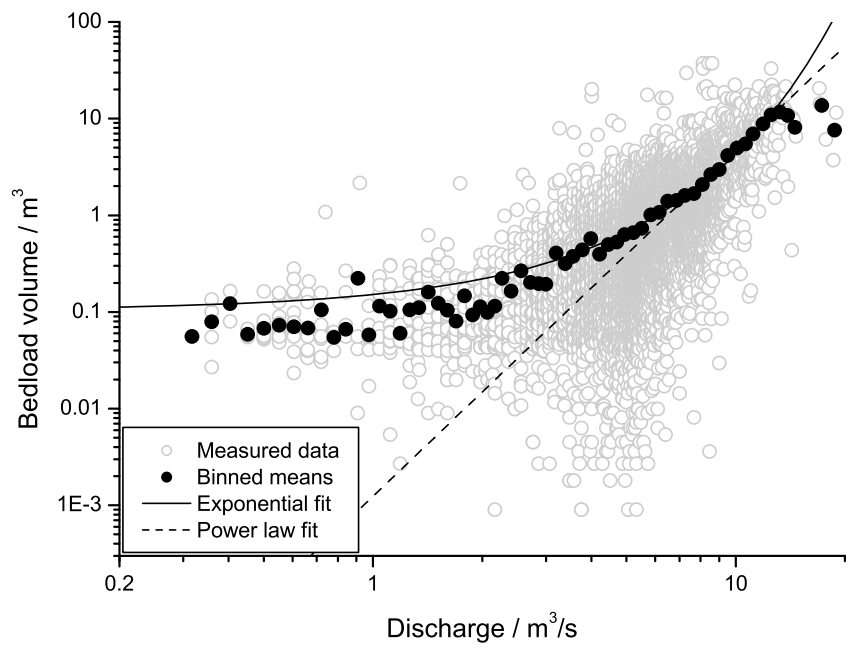

Figure 2. Bed load volume accumulated in 15 min periods as function of discharge. Volumes smaller than $\sim 0.05 \mathrm{~m}^{3}$ are often missed by the instruments and are thus underrepresented. Exponential and power law fits were done on binned means (black circles), instead of on the whole set. Fit values are given in the caption of Figure 4. 
Probability density pdf
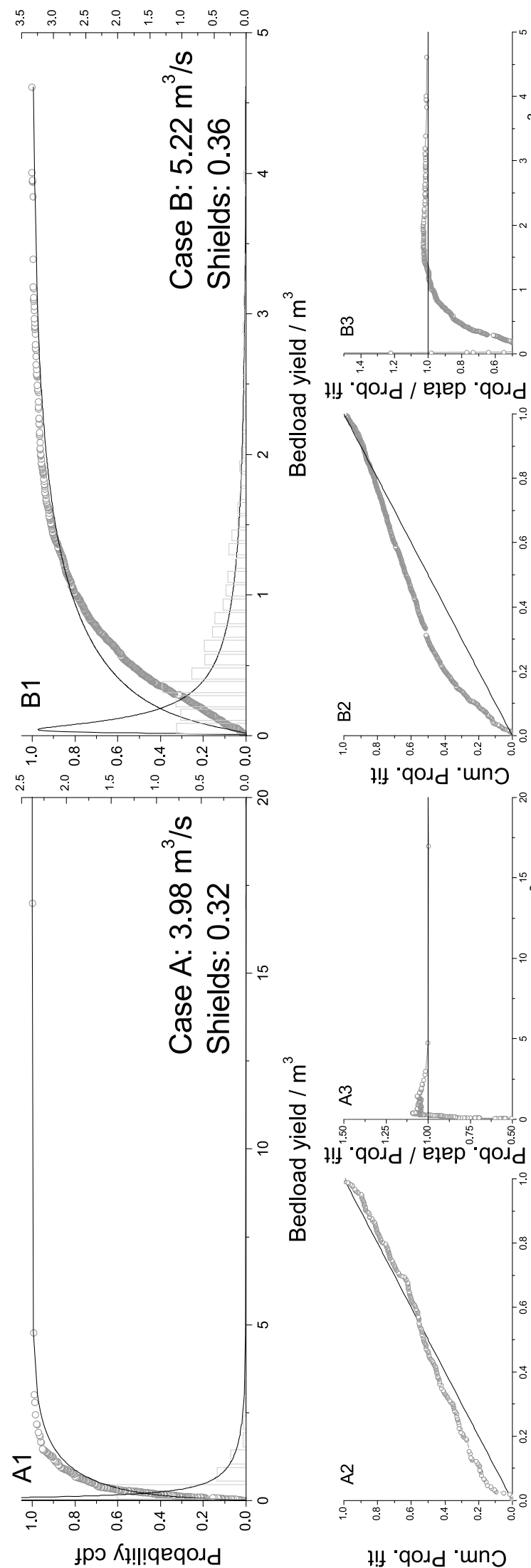

Probability density pdf
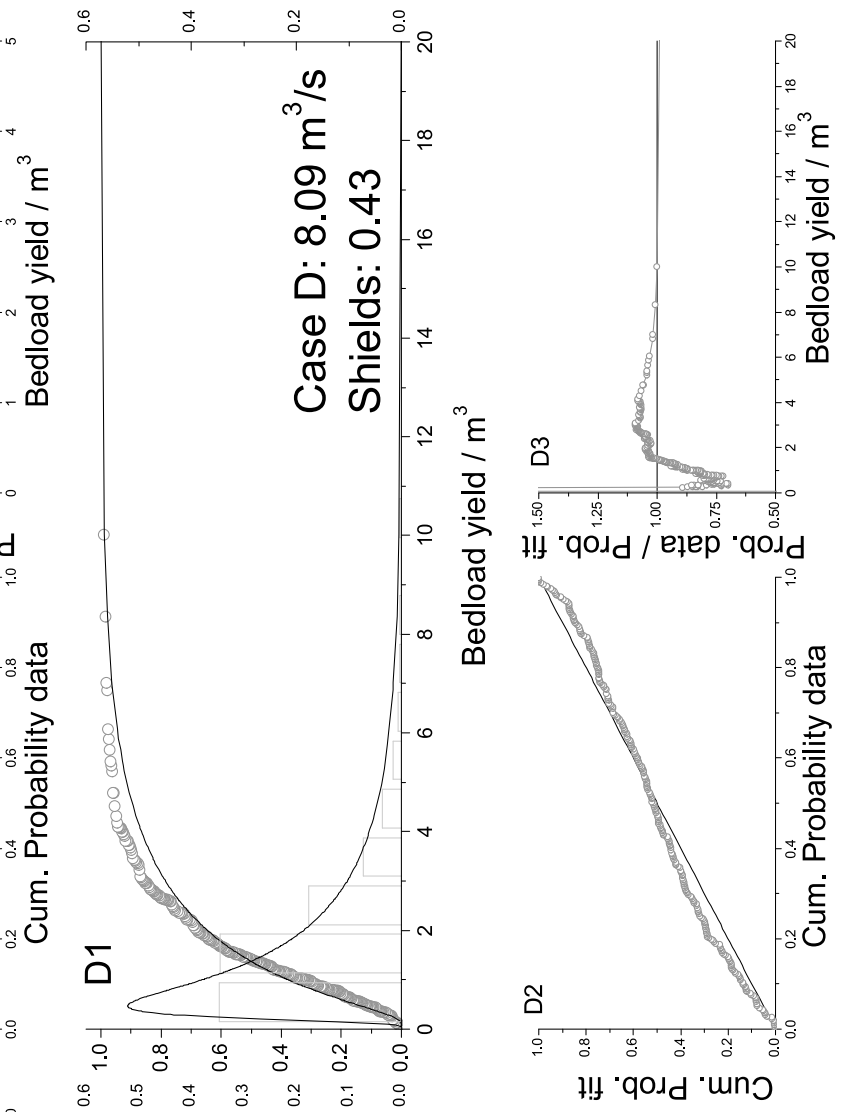

I!f 'qoud 'unj

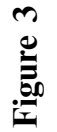



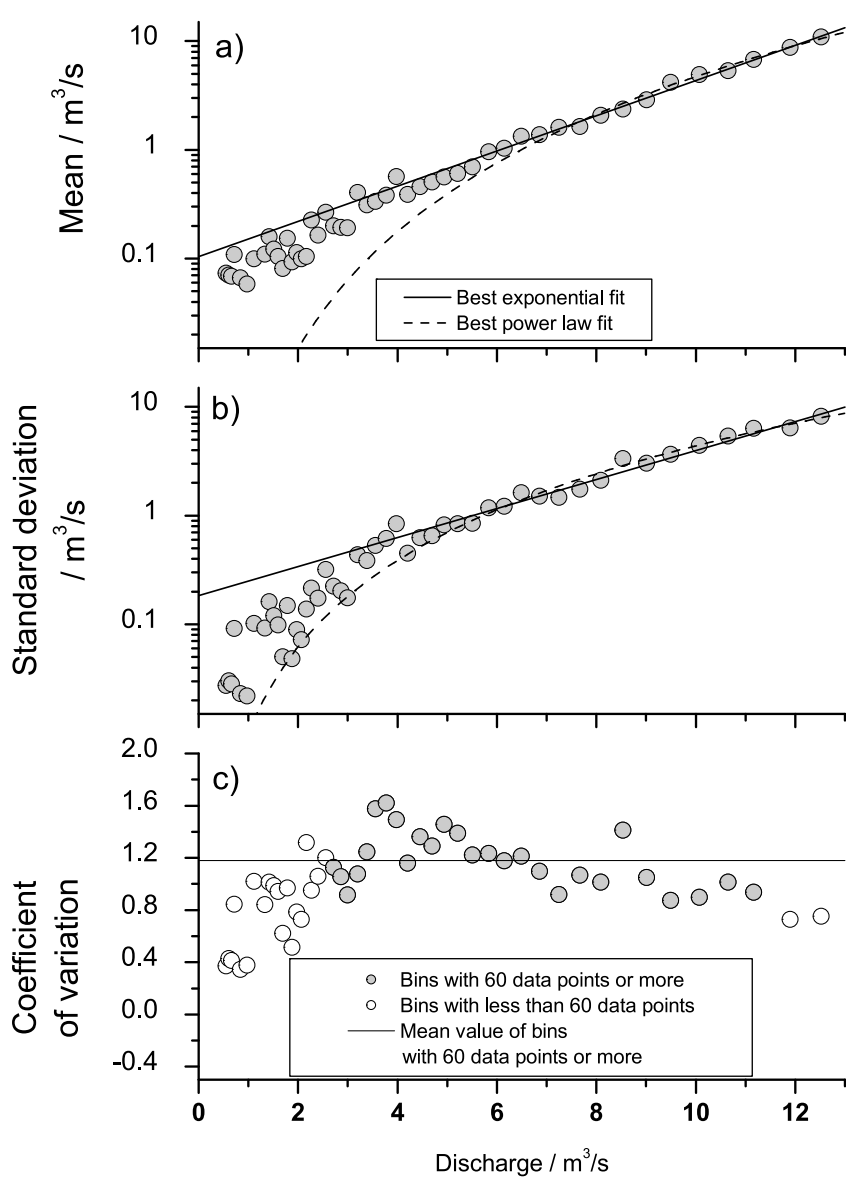

Figure 4. Variation of the best fit mean value and the standard deviation with discharge for each bin with at least 15 data points. The coefficient of variation is approximately constant for all bins with at least 80 data points. The fit values for the exponential $y=A \exp (B Q)$ and the power law $y=a Q^{b}$ are for the mean value in (a) $A=0.081 \mathrm{~m}^{3} / \mathrm{s}$, $B=0.40 \mathrm{~s} / \mathrm{m}^{3}, a=0.0012\left(\mathrm{~m}^{3} / \mathrm{s}\right)^{1-b}$, and $b=3.58$, and for the standard deviation in (b) $A=0.18 \mathrm{~m}^{3} / \mathrm{s}, B=0.31 \mathrm{~s} / \mathrm{m}^{3}$, $a=0.0099\left(\mathrm{~m}^{3} / \mathrm{s}\right)^{1-b}$, and $b=2.65$.

directly related to discharge at a single location) for bed load transport rates at Goodwin Creek. Although this relationship needs to be confirmed for other streams, the assumption $\operatorname{std}\left(Q_{\mathrm{s}}\right) \propto \mathrm{E}\left(Q_{\mathrm{s}}\right)$ may be a good first approximation for the standard deviation.

\subsection{Comparison to Other Distribution Functions}

[18] Next, the Pitzbach data is compared to other distributions. In addition to a continuous form of the Poisson distribution (equation (15)) and the gamma distribution (equation (19)), the distributions due to Hamamori [1962] and Carey and Hubbell [1986] are tested, which were derived for bed form-dominated transport. The Hamamori distribution is valid in the range from zero to four times the mean transport rate $Q_{\mathrm{m}}$ and has a pdf of the form

$$
\operatorname{pdf}\left(Q_{\mathrm{s}}\right)=\frac{1}{4 Q_{\mathrm{m}}} \ln \left\{\frac{4 Q_{\mathrm{m}}}{Q_{\mathrm{s}}}\right\}
$$

The cdf has the form

$$
\operatorname{cdf}\left(Q_{\mathrm{s}}\right)=\frac{Q_{\mathrm{s}}}{4 Q_{\mathrm{m}}}\left(1+\ln \left\{\frac{4 Q_{\mathrm{m}}}{Q_{\mathrm{s}}}\right\}\right) .
$$

The distribution is top bounded, i.e., transport rates with values larger than four times $Q_{\mathrm{m}}$ are assigned a probability of zero. Carey and Hubbell [1986] generalized the model and derived the pdf

$$
\operatorname{pdf}\left(Q_{\mathrm{s}}\right)=\frac{Q_{\max }^{1 / n-1}-\left(Q_{\mathrm{s}} / Q_{\mathrm{m}}\right)^{1 / n-1}}{(1-n) Q_{\max }^{1 / n}} .
$$

Here $n$ is a constant, which is generally smaller than one [Gomez et al., 1989], and the maximum possible transport rate $Q_{\max }$ is given by

$$
Q_{\max }=2(n+1) Q_{\mathrm{m}}
$$

The cdf corresponding to the pdf in equation (22) is given by

$$
\operatorname{pdf}\left(Q_{\mathrm{s}}\right)=\frac{\left(Q_{\mathrm{s}} / Q_{\mathrm{m}}\right)}{(1-n) Q_{\max }}-\frac{n\left(Q_{\mathrm{s}} / Q_{\mathrm{m}}\right)^{1 / n}}{(1-n) Q_{\max }^{1 / n}} .
$$

Similarly to the Hamamori distribution, the Carey-Hubbell distribution is top bounded at $Q_{\max }$. In the Pitzbach, maximum transport rates exceed mean transport rates by a factor of four at most discharges. Thus, the Hamamori and CareyHubbell distributions are clearly of limited value to describe the data. However, in the range for which they are valid, both models give a reasonable fit to the data (Figure 5). With a value of $n=0.5$, the Carey-Hubbell distribution closely traces the data for low transport rates (below about the 50 percentile; Figure 5c), while the Hamamori distribution fits well over the whole range of its validity.

[19] Both the continuous Poisson and the gamma distribution give better fits to the data than the Birnbaum-Saunders distribution for low transport rates. The gamma distribution gives a good fit for the whole data range, with maximum deviations of $\sim 5 \%$. All three distributions (BirnbaumSaunders, continuous version of the Poisson distribution,

Figure 3. Cumulative probability distribution and probability density functions (large figures labeled A1, etc.) of the observed loads (open circles, histogram) and the best fit (solid line) using equation (7) for discharges of (a) $3.98 \mathrm{~m}^{3} / \mathrm{s}$ (292 data points), (b) $5.22 \mathrm{~m}^{3} / \mathrm{s}$ (1223 data points), (c) $6.49 \mathrm{~m}^{3} / \mathrm{s}$ (477 data points), and (d) $8.09 \mathrm{~m}^{3} / \mathrm{s}$ (335 data points). The corresponding Shields number estimated for the artificial cross section at the measurement site is given on the plot. Small figures show (left, 2) percent-percent plots and (right, 3) probability-ratio plots for the same discharges. Percent-percent plots allow a good optical evaluation of the fit in the lower percentiles, while ratio plots allow the evaluation of the fits in the right-hand tail. 

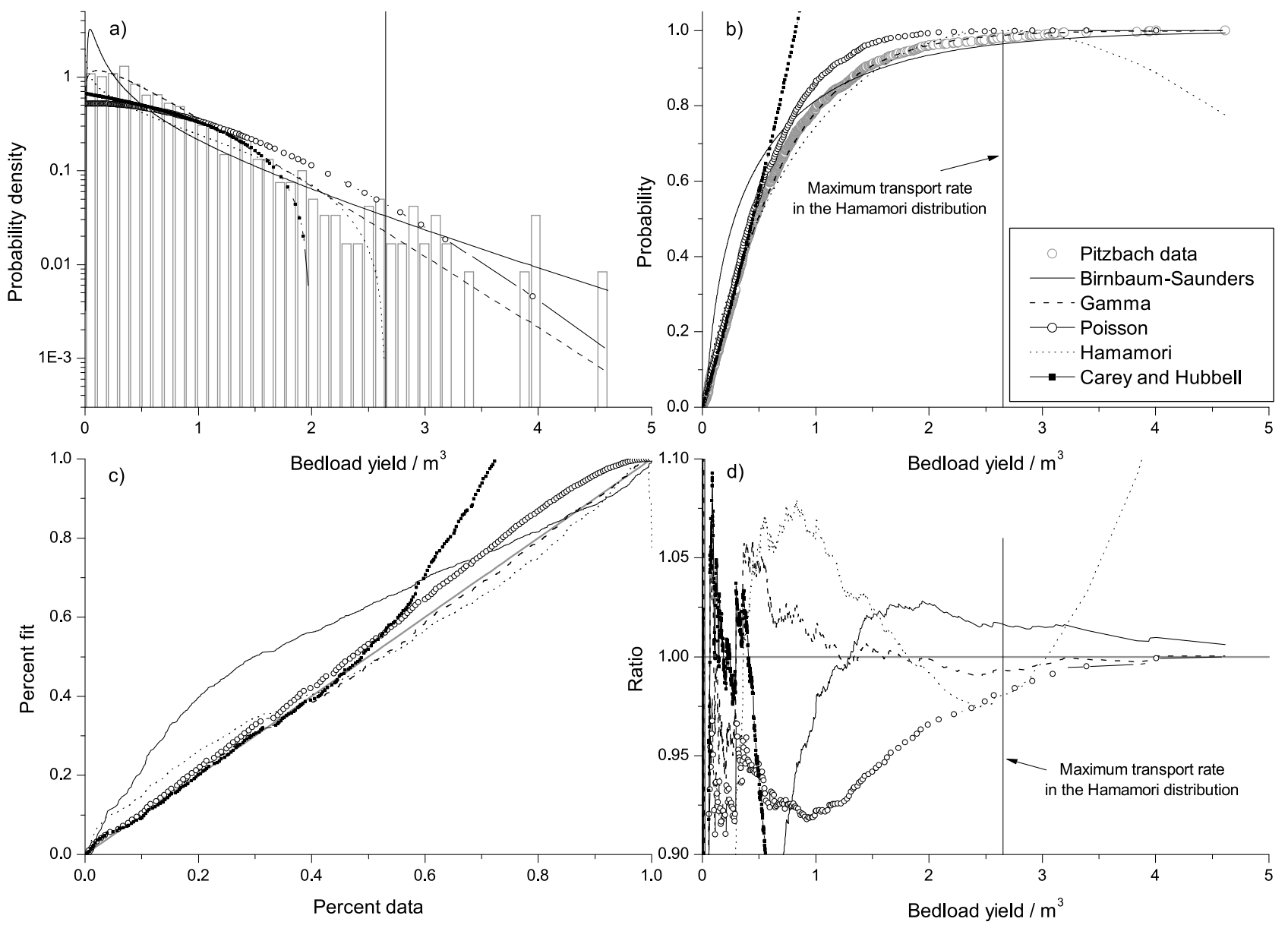

Figure 5. Comparison of the Hamamori, Carey-Hubbell, Birnbaum-Saunders, gamma, and Poisson distributions to the Pitzbach data at a discharge of $5.22 \mathrm{~m}^{3} / \mathrm{s}$ (1223 data points). (a) Probability density functions on a semilogarithmic plot. (b) Cumulative probability functions. (c) Percent-percent plots. This visualization allows an assessment of the goodness of fit for low and medium transport rates. (d) Percentileratio plots. This visualization allows the assessment of the goodness of fit in the tail region. The gamma distribution gives the best fit with maximum deviations of $\sim 5 \%$. The Birnbaum-Saunders distribution provides a reasonable approximation especially to the right-hand tail.

gamma) converge onto the right-hand tail at high transport rates (Figure 5d).

\section{Discussion}

\subsection{The Birnbaum-Saunders Distribution}

[20] The assumptions made in the derivation of the Birnbaum-Saunders distribution warrant a discussion of the generality of the function. Since the argument is purely statistical, the function is independent of the physics of sediment transport and should be widely applicable. However, the applicability is restricted to systems where individual particle arrivals are countable and statistically independent, and the function may not be applicable in environments where transport is dominated by bed form motion, as in many sand-bed streams. In such environments, distribution functions specifically developed for dune motion, such as the Hamamori or the Carey-Hubbell distributions, may yield better results (see for example the work of Carey [1985] for field testing of the Hamamori distribution in a sand-bed river). It may be possible to adapt the derivation of the Birnbaum-Saunders distribution by not considering the arrival of individual particles but the arrival of individual bed forms. The final distribution of transport rates would then have the same form. However, the assumptions in the derivation set a constraint on the length of the measurement interval needed to make the distribution applicable to a data set: it needs to be long enough such that the number of particles arriving within it is large enough such that the central limit theorem and the law of large numbers apply. In natural channels discharge can fluctuate quickly and it may not be easy to find a suitable measurement interval that ensures that hydraulic conditions are approximately constant while a sufficient number of bed forms arrive.

[21] The constraint on the measurement interval implies that at small transport rates, i.e., when only few particles arrive, the Birnbaum-Saunders distribution will necessarily break down. This may be one of the reasons for the unsatisfactory fit of the function to the Pitzbach data for small transport rates (cf. Figure 3). The rate of convergence to the normal distribution in the central limit theorem can be 
quantified with the Berry-Esséen Theorem [Berry, 1941; Esséen, 1942], which states that the maximum difference between the real distribution and its normal approximation scales with $N^{-1 / 2}$. Thus, the approximation gets better as more particles are trapped within the measurement interval. In general, the error depends on the mean interarrival time $\mu$ and varies with discharge. If the underlying distribution is exponential (section 2.2), the construction of a distribution function from added random numbers is reasonably approximated by a normal distribution for $N>100$ for the conditions at the Pitzbach. For comparison, assuming that the mean grain size of the material deposited in the retention basin at the Pitzbach is close to the 65 percentile of the size distribution of $6 \mathrm{~mm}$ [Rickenmann and McArdell, 2008], around 2 million particles are present in $1 \mathrm{~m}^{3}$ of bed load, assuming $50 \%$ pore space and $50 \%$ fines. A volume of $1 \mathrm{~m}^{3}$ is close to the average yield delivered within $15 \mathrm{~min}$ at a discharge of $8 \mathrm{~m}^{3} / \mathrm{s}$ (Figure 2). Thus, for most practical conditions, the rate of convergence to the normal distribution should not be a problem.

[22] The assumption of the law of large numbers leads to similar problems. At the Pitzbach, the mean particle diameter is around $6 \mathrm{~mm}$ for the material deposited in the retention basin. At small transport rates often large grain sizes are underrepresented in the bed load [Wilcock and McArdell, 1993], and for the Pitzbach there is no information available on how the grain size distribution (and the mean grain size) changes with discharge and bed load transport rate. An accurate quantification of the rate of convergence is thus not currently possible; however, considering the large number of arriving particles, the measured mean should be fairly close to the population mean.

[23] In addition to these statistical errors, at small transport rates the instruments are unreliable, which affects goodness of fit. Often small volume changes are missed by the equipment and data in this range is underrepresented in the distribution. One can get around these problems by just extending the measurement intervals. However, again it may be problematic to find a suitable interval in which the variation of discharge is slow enough such that the hydraulic conditions can be assumed to be constant.

[24] In the Birnbaum-Saunders distribution the mean transport rate decreases with increasing sampling interval (see equation (8)). Similarly, both in the field [Bunte and $A b t, 2005]$ and in the laboratory [Singh et al., 2009], it was found that at low transport rates the estimated mean transport rates decrease as the sampling interval increases, while at high transport rates the trend reverses. Singh et al. [2009] related this trend reversal to the presence of large bed forms and their dominance in the transport process at high transport rates. As discussed above, the assumptions underlying the Birnbaum-Saunders distribution are not valid when transport is dominated by bed form motion, and thus the predictions can be considered to be in line with observations. The Pitzbach data span 2 years, and during this time no exceptional flood occurred in the catchment. Sufficient data for the construction of a distribution function is available for discharges up to $\sim 10 \mathrm{~m}^{3} / \mathrm{s}$, whereby the estimated peak discharge of a 2-year flood is $18-20 \mathrm{~m}^{3} / \mathrm{s}$. Consequently, the Birnbaum-Saunders distribution cannot currently be tested under high discharge conditions. Singh et al. [2009] also described multiscaling of the moments of bed load transport for different sampling intervals, i.e., different moments such as mean and standard deviation are dependent on the sampling interval in different ways. This behavior is predicted by the Birnbaum-Saunders distribution (cf. equations (8) and (9)).

\subsection{The Pitzbach Data and Comparison With Other Distribution Functions}

[25] Bed load volumes measured at the Pitzbach within 15-min periods scatter widely, over up to four orders of magnitude at a given discharge (Figure 2). It is clear that nonlinear regression with a power law, as is often used to analyze field data of sediment transport, is inadequate to describe observations. First, regression always gives a global optimization and can lead to strong local deviations. Second, the large scatter in the $y$ direction is treated as a measurement error and not as a genuine signal. Herein, a binning procedure was used to analyze the data. This has the advantage that statistics for transport rates at a given discharge can be calculated for each bin, and one can obtain information not only on the mean behavior but also on the standard deviation and the shape of the probability distributions.

[26] From the tested distributions (Birnbaum-Saunders, continuous Poisson-type distribution, gamma, Hamamori, Carey-Hubbell), the gamma distribution gives the best fit to the Pitzbach data. Both Birnbaum-Saunders and continuous Poisson distributions provide reasonable fits in the righthand tail, while the Hamamori and Carey-Hubbell distributions fit well for low transport rates. Few other data sets exist with a sufficient size and quality to make similar calculations as done here for the Pitzbach. Kuhnle and Willis [1998] tested the exponential, the normal, the gamma, and the Hamamori distributions for bed load transport data from Goodwin Creek and likewise found that the gamma distribution gives the best description of the data. Thus, the gamma distribution has been observed to work well at two field sites and can be recommended for use in field applications at the current state of knowledge. However, the Birnbaum-Saunders distribution may be more suitable in certain applications, because its derivation is more general. After all, the gamma distribution was derived from the assumption of Poisson-distributed interarrival times. The precise form of the distribution of interarrival times has not been measured in the field and is debated from a theoretical point of view. For the Pitzbach data, the tail behavior of sediment transport rates at a given discharge is well described by the Birnbaum-Saunders distribution for the upper twentieth percentile, at many discharges the good fit region reaches to the upper thirtieth percentile or further. The upper twentieth percentile corresponds to $60 \%-70 \%$ of the total load transported for a given discharge. The distribution may therefore provide an adequate approximation in many instances.

[27] The Birnbaum-Saunders and the gamma distribution decline exponentially in $Q_{\mathrm{s}}$ in the limit of large transport rates. Hence, large events occur more commonly as would be expected when using a normal distribution. Environmental parameters often show a power law tail (heavy tail), for example landslide sizes or stream discharge [e.g., Stark and Hovius, 2001; Lague et al., 2005], and it is some- 
times assumed that sediment transport rates likewise are heavy tailed. On the contrary, in the Pitzbach bed load transport rates show an exponential rather than a power law tail. Large events are thus not as dominant for the total transport rate as is observed for many other environmental processes. Note, however, that this is only true for transport rates at a given discharge. The long-term sediment budget of a catchment may be more dependent on the distribution of flood sizes (often heavy tailed) than on the properties of the distributions considered herein.

\section{Conclusions}

[28] Bed load transport rates are known to fluctuate strongly even under steady hydraulic conditions. Here, I have shown a new way of deriving probability distributions of bed load transport rates by considering the waiting times between the arrivals of individual particles (interarrival times). The assumptions underlying the derivation should make the distributions applicable for bed load transport not dominated by bed form motion. Without any further assumptions about the distribution of interarrival times, the formalism yields the Birnbaum-Saunders distribution as a general approximation. A continuous version of the Poisson distribution and the gamma distribution can be derived if the interarrival times are assumed to be exponentially or Poisson distributed, respectively. The approach circumvents the need for calibration of several parameters that are hard to observe directly, as is often necessary in modern stochastic theories of sediment transport. In fact, with modern equipment such as light tables and video-based observation [Drake et al., 1988; Frey et al., 2003; Zimmermann et al., 2008], it should be possible to directly measure the distribution of interarrival times in the field or the laboratory.

[29] Together with the previously proposed Hamamori and Carey-Hubbell distributions [Hamamori, 1962; Carey and Hubbell, 1986], the derived functions were tested on a large data set from the Pitzbach, Austria. Out of the tested distributions, the gamma distribution performs best, with maximal deviations from the data of around $5 \%$. The Birnbaum-Saunders distribution provides a reasonable approximation especially in the right-hand tail. The Poisson distribution fits well over the whole range of the data. The Hamamori and Carey-Hubbell distributions give reasonable fits especially for low transport rates. The gamma distribution has previously been found to also describe the Goodwin Creek data well [Kuhnle and Willis, 1998]. At the current state of knowledge, the gamma distribution can thus be recommended for use in field applications. However, the Birnbaum-Saunders distribution is a reasonable approximation, which has been derived without making any debated physical assumptions. Thus, its use may be advantageous in certain applications.

\section{Notation}

Variables

$M$ Stochastic variable of mass, $\mathrm{kg}$

$m$ Total mass of $N$ particles, $\mathrm{kg}$

$\bar{m}$ Average mass of a particle, $\mathrm{kg}$

$N$ Number of particles

$n$ Carey-Hubbell exponent

$Q_{\mathrm{m}}$ Mean bed load mass flux, $\mathrm{kg} / \mathrm{s}$
$Q_{\max }$ Maximum bed load mass flux in the CareyHubbell distribution, $\mathrm{kg} / \mathrm{s}$

$Q_{\text {s }}$ Bed load mass flux, $\mathrm{kg} / \mathrm{s}$

$T$ Stochastic variable of waiting time, $\mathrm{s}$

$T_{N}$ Time until the arrival of the $N$ th particle, $\mathrm{s}$

$t$ Length of measurement interval, $\mathrm{s}$

$t_{N}$ Stochastic variable of the time until the arrival of the $N$ th particle, $\mathrm{s}$

$x, y, z$ Variables, used variously

$\beta, \beta^{\prime}$ Scale factor in the Birnbaum-Saunders distribution, $\mathrm{kg}$ (unprimed), $\mathrm{kg} / \mathrm{s}$ (primed)

$\gamma$ Shape factor in the Birnbaum-Saunders distribution

$\mu$ Mean waiting time between individual particle arrivals, $\mathrm{s}$

$\sigma_{w}$ Standard deviation of waiting times between individual particle arrivals, $\mathrm{s}$

\section{Functions}

$\operatorname{cdf}(x)$ Cumulative distribution function of $x$

$\operatorname{cva}(x)$ Coefficient of variation of $x$

$\mathrm{E}(x)$ Expectation values of $x$

$\operatorname{pdf}(x)$ Probability density function of $x$

$\operatorname{std}(x)$ Standard deviation of $x$

$\operatorname{var}(x)$ Variance of $x$

$\Gamma(x), \Gamma(x, y)$ Complete and upper incomplete Gamma function

$\Phi((x-\mu) / \sigma)$ Cumulative normal distribution of $x$ with mean $\mu$ and standard deviation $\sigma$

[30] Acknowledgments. Many people have commented on the ideas presented herein and have helped to shape them in discussions. I thank especially D. Rickenmann, A. Badoux, J.W. Kirchner, B.W. McArdell, M. Nitsche, D. Lague, and M. Stähli for support, encouragement, stimulating discussions, and comments on earlier versions of the manuscript. TIWAG supported the measurement campaign at the Pitzbach and made the data available. A. Recking, C. Ancey, and an anonymous reviewer commented on an earlier version of the manuscript.

\section{References}

Ancey, C., T. Böhm, M. Jodeau, and P. Frey (2006), Statistical description of sediment transport experiments, Phys. Rev. E, 74, 011302 , doi:10.1103/PhysRevE.74.011302.

Ancey, C., A. C. Davison, T. Böhm, M. Jodeau, and P. Frey (2008), Entrainment and motion of coarse particles in a shallow water stream down a steep slope, J. Fluid Mech., 595, 83-114, doi:10.1017/ S0022112007008774.

Benda, L., and T. Dunne (1997), Stochastic forcing of sediment routing and storage in channel networks, Water Resour. Res., 33(12), 2865-2880, doi:10.1029/97WR02387.

Berry, A. C. (1941), The accuracy of the Gaussian approximation to the sum of independent variates, Trans. Am. Math. Soc., 49, 122-136.

Birnbaum, Z. W., and S. C. Saunders (1968), A probabilistic interpretation of Miner's rule, SIAM J. Appl. Math., 16(3), 637-652.

Bunte, K., and S. R. Abt (2005), Effect of sampling time on measured gravel bed load transport rates in a coarse-bedded stream, Water Resour. Res., 41, W11405, doi:10.1029/2004WR003880.

Carey, W. P. (1985), Variability in measured bed load-transport rates, Water Resour. Bull., 21, 39-48.

Carey, W. P., and D. W. Hubbell (1986), Probability distributions for bed load transport, in Proceedings of the 4th Federal Inter-Agency Sediment Conference, US Geological Survey, vol. 2, 4131-4140.

Chen, L., and M. C. Stone (2008), Influence of bed material size heterogeneity on bed load transport uncertainty, Water Resour. Res., 44, W01405, doi:10.1029/2006WR005483.

Desmond, A. (1985), Stochastic models of failure in random environments, Can. J. Stat., 13(2), 171-183.

Drake, T. G., R. L. Shreve, W. E. Dietrich, P. J. Whiting, and L. B. Leopold (1988), Bed load transport of fine gravel observed by motion-picture photography, J. Fluid Mech., 192, 193-217. 
Einstein, H. A. (1937), Der Geschiebetrieb als Wahrscheinlichkeitsproblem, Mitt. Versuchsanst. Wasserbau Eidg. Tech. Hochsch. Zürich, Rascher, Zürich, Switzerland.

Esséen, C. G. (1942), On the Liapounoff Limit of Error in the theory of probability, Ark. Mat. Astr. Och Fys., 28A(9), 1-19.

Frey, P., C. Ducottet, and J. Jay (2003), Fluctuations of bed load solid discharge and grain size distribution on steep slopes with image analysis, Exp. Fluids, 35(6), 589-597, doi:10.1007/s00348-003-0707-9.

Gomez, B., and M. Church (1989), An assessment of bed load transport formulae for gravel bed rivers, Water Resour. Res., 25(6), 1161-1186.

Gomez, B., R. L. Naff, and D. W. Hubbell (1989), Temporal variations in bed load transport rates associated with the migration of bed forms, Earth Surf. Processes Landforms, 14, 135-156.

Hamamori, A. (1962), A theoretical investigation on the fluctuations of bed load transport, Rep. R4, Delft Hydraulics Laboratory, Delft, Netherlands.

Hassan, M. A., and M. Church (2000), Experiments on surface structure and partial sediment transport on a gravel bed, Water Resour. Res., 36, 1885-1895, doi:10.1029/2000WR900055.

Hoey, T. (1992), Temporal variations in bed load transport rates and sediment storage in gravel bed rivers, Prog. Phys. Geog., 16, 319-338.

Hofer, B. (1987), Der Feststofftransport von Hochgebirgsbächen am Beispiel des Pitzbachs, Österreichische Wasserwirtschaft, 39, 30-38.

Johnson, N. L., S. Kotz, and N. Balakrishnan (1995), Continuous univariate distributions, 2nd ed., vol. 2, pp. 651-663, John Wiley, New York.

Kirchner, J. W., W. E. Dietrich, F. Iseya, and H. Ikeda (1990), The variability of critical shear Stress, friction angle, and grain protrusion in waterworked sediments, Sedimentology, 37, 647-672.

Kuhnle, R. A., and J. B. Southard (1988), Bed load transport fluctuations in a gravel bed laboratory channel, Water Resour. Res., 24, 247-260.

Kuhnle, R. A., and J. C. Willis (1998), Statistics of sediment transport in Goodwin Creek, J. Hydrol. Eng., 124, 1109-1114.

Lague, D., N. Hovius, and P. Davy (2005), Discharge, discharge variability, and the bedrock channel profile, J. Geophys. Res., 110, F04006, doi:10.1029/2004JF000259.

Lisle, I. G., C. W. Rose, W. L. Hogarth, P. B. Hairsine, G. C. Sander, and J. Parlange (1998), Stochastic sediment transport in soil erosion, J. Hydrol., 204, 217-230.
Lisle, T. E., Y. Cui, G. Parker, J. E. Pizzuto, and A. M. Dodd (2001), The dominance of dispersion in the evolution of bed material waves in gravel bed rivers, Earth Surf. Processes Landforms, 26, 1409-1420, doi:10.1002/esp.300.

Papanicolaou, A. N., P. Diplas, N. Evaggelopoulos, and S. Fotopoulos (2002), Stochastic incipient motion criterion of spheres under various bed packing conditions, J. Hydrol. Eng., 128(4), 369-380, doi:10.1061/ (ASCE)0733-9429(2002)128:4(369).

Recking, A., P. Frey, A. Paquier, and P. Belleudy (2009), An experimental investigation of mechanisms involved in bed load sheet production and migration, J. Geophys. Res., 114, F03010, doi:10.1029/2008JF000990.

Rickenmann, D., and B. W. McArdell (2008), Calibration of piezoelectric bed load impact sensors in the Pitzbach mountain stream, Geodin. Acta, 21, 35-52, doi:10.3166/ga.21.35-52.

Singh, A., K. Fienberg, D. J. Jerolmack, J. Marr, and E. Foufoula-Georgiou (2009), Experimental evidence for statistical scaling and intermittency in sediment transport rates, J. Geophys. Res., 114, F01025, doi:10.1029/ 2007JF000963.

Stark, C. P., and N. Hovius (2001), The characterization of landslide size distributions, Geophys. Res. Lett., 28, 1091-1094.

Turowski, J. M. (2009), Stochastic modeling of the cover effect and bedrock erosion, Water Resour. Res., 45, W03422, doi:10.1029/ 2008WR007262.

Turowski, J. M., and D. Rickenmann (2009), Tools and cover effects in bed load transport observations in the Pitzbach, Austria, Earth Surf. Processes Landforms, 34, 26-37, doi:10.1002/esp.1686.

Wilcock, P. R., and B. W. McArdell (1993), Surface-based fractional transport rates: Mobilization thresholds and partial transport of a sand-gravel sediment, Water Resour. Res., 29(4), 1297-1312.

Willis, J. C., and G. C. Bolton (1979), Statistical analysis of concentration records, J. Hydrol. Div., 105(1), 1-15.

Zimmermann, A., M. Church, and M. A. Hassan (2008), Video-based gravel transport measurements with a flume mounted light table, Earth Surf. Processes Landforms, 33, 2285-2296, doi:10.1002/esp.1675.

J. M. Turowski, Eidgenössische Forschungsanstalt WSL Birmensdorf, Zürcherstrasse 111,8903 Birmensdorf, Switzerland. (jens.turowski@wsl.ch) 